Pacific

Journal of

Mathematics

CONNECTIVITY OF FINITE SUBSET SPACES OF CELL COMPLEXES

Christopher Tuffley

Volume $217 \quad$ No. 1

November 2004 


\title{
CONNECTIVITY OF FINITE SUBSET SPACES OF CELL COMPLEXES
}

\author{
Christopher Tuffley
}

\begin{abstract}
The $k$-th finite subset space of a topological space $X$ is the space $\exp _{k} X$ of nonempty subsets of $X$ of size at most $k$, topologised as a quotient of $X^{k}$. Using results from our earlier paper on the finite subset spaces of connected graphs we show that the $k$-th finite subset space of a connected cell complex is $(k-2)$-connected, and $(k-1)$-connected if in addition the underlying space is simply connected. We expect $\exp _{k} X$ to be $(k+m-2)$-connected if $X$ is an $m$-connected cell complex, and reduce proving this to the problem of proving it for finite wedges of $(m+1)$-spheres. Our results complement a theorem due to Handel that for path-connected Hausdorff $X$ the map on $\pi_{i}$ induced by the inclusion $\exp _{k} X \hookrightarrow \exp _{2 k+1} X$ is zero for all $\mathrm{k} \geq 1$ and $i \geq 0$.
\end{abstract}

\section{Introduction}

The $k$-th finite subset space of a topological space $X$ is the $\operatorname{space}^{\exp _{k}} X$ of nonempty subsets of $X$ of size at most $k$, topologised as a quotient of $X^{k}$ via the map

$$
\left(x_{1}, \ldots, x_{k}\right) \mapsto\left\{x_{1}\right\} \cup \cdots \cup\left\{x_{k}\right\} .
$$

The construction is functorial, with $f: X \rightarrow Y$ inducing $\exp _{k} f: \exp _{k} X \rightarrow$ $\exp _{k} Y$ sending $\alpha \subseteq X$ to $f(\alpha) \subseteq Y$, and moreover $\exp _{k} f$ and $\exp _{k} g$ are homotopic if $f$ and $g$ are, so that $\exp _{k}$ is in fact a homotopy functor.

Handel [3] has shown that for path-connected Hausdorff $X$ the map on $\pi_{i}$ induced by the inclusion $\exp _{k} X \hookrightarrow \exp _{2 k+1} X$ is zero. Using results from our paper [6] on the finite subset spaces of connected graphs we complement this result, proving the following connectivity theorem for the finite subset spaces of a connected cell complex:

Theorem 1. The $k$-th finite subset space of a connected cell complex $X$ is $(k-2)$-connected, in other words $\pi_{i}\left(\exp _{k} X\right)$ vanishes for $i \leq k-2$.

Using results from [7], if $X$ is a simply connected cell complex, then the conclusion may be strengthened to $\exp _{k} X$ is $(k-1)$-connected. We 
expect further that $\exp _{k} X$ should be $(k+m-2)$-connected if $X$ is an $m$ connected cell complex. We discuss this in Section 3 after proving the result in Section 2.

\section{Proof of Theorem 1}

We note that Theorem 1 is immediate for $k=2$, since the finite subset spaces of a path-connected space are again path-connected, so in proving it we assume $k \geq 3$. In this case the conclusion is equivalent to the statement that $\exp _{k} X$ is simply connected with vanishing reduced homology in dimensions less than $k-1$, by the Hurewicz theorem, and we shall freely use this formulation. We first prove the result for finite complexes and then pass to the infinite case via a compactness argument.

Proof of Theorem 1 for finite $X$. The proof is by induction on the dimension of $X$, with the base case $\operatorname{dim} X=1$ given by our paper [6], in which we studied the finite subset spaces of connected finite graphs. The keys to the inductive step are the following two observations:

(1) If $P_{1}, \ldots, P_{k+1}$ are disjoint subsets of $X$ then a $k$ element subset of $X$ must lie in $X \backslash P_{\ell}$ for some $\ell$.

(2) If $X$ is a connected finite $(n+1)$-complex, $n \geq 1$, and $P \subseteq X$ lies in the open $(n+1)$-cells and intersects each in a nonempty finite set, then $X \backslash P$ has the homotopy type of a connected finite $n$-complex.

We use these in conjunction with the following lemma, itself proved inductively using the Mayer-Vietoris sequence.

Lemma 1. Let $Y$ be a union of open sets $U_{1}, \ldots, U_{r}$ such that $U_{1} \cap \cdots \cap U_{r}$ is nonempty and each $U_{i_{1}} \cap \cdots \cap U_{i_{s}}$ has vanishing reduced homology in dimensions less than $j$. Then $Y$ has vanishing reduced homology in dimensions less than $j$ also.

Suppose the theorem holds for connected finite $n$-complexes, for some $n \geq 1$, and let $X$ be a connected finite $(n+1)$-complex. Let $v$ be a vertex of $X$ and let $P_{1}, \ldots, P_{k+1}$ be disjoint subsets of $X$ each consisting of exactly one point from each open $(n+1)$-cell. By observation (1) the sets

$$
\mathcal{U}_{\ell}=\exp _{k}\left(X \backslash P_{\ell}\right)
$$

cover $\exp _{k} X$, and each $\mathcal{U}_{\ell}$ is open since $\left(X \backslash P_{\ell}\right)^{k}$ is open in $X^{k}$. Moreover the intersection $\mathcal{U}_{1} \cap \cdots \cap \mathcal{U}_{k+1}$ contains $\{v\}$ and is therefore nonempty.

Consider

$$
\mathcal{U}_{\ell_{1}} \cap \cdots \cap \mathcal{U}_{\ell_{s}}=\exp _{k}\left(X \backslash\left(P_{\ell_{1}} \cup \cdots \cup P_{\ell_{s}}\right)\right) .
$$

By observation (2) the space $X \backslash\left(P_{\ell_{1}} \cup \cdots \cup P_{\ell_{s}}\right)$ has the homotopy type of a connected finite $n$-complex, so by the inductive hypothesis $\mathcal{U}_{\ell_{1}} \cap \cdots \cap \mathcal{U}_{\ell_{s}}$ has vanishing reduced homology in dimensions less than $k-1$. It follows that 
the hypotheses of Lemma 1 are satisfied by the cover $\left\{\mathcal{U}_{\ell}\right\}$ with $j=k-1$, and we conclude that $\exp _{k} X$ has vanishing reduced homology in dimensions less than $k-1$.

To complete the inductive step it remains to show that $\exp _{k} X$ is simply connected. This follows immediately from the van Kampen theorem applied to the cover $\left\{\mathcal{U}_{\ell}\right\}$ with basepoint $\{v\}$. By the inductive hypothesis we have $\pi_{1}\left(\mathcal{U}_{\ell},\{v\}\right) \cong\{1\}$ for all $\ell$, and since each $\mathcal{U}_{j} \cap \mathcal{U}_{\ell}$ is path-connected we get immediately $\pi_{1}\left(\exp _{k} X,\{v\}\right) \cong\{1\}$ also.

To pass to the infinite-dimensional case we use the following lemma, which we prove with no assumptions on $X$.

Lemma 2. If $C \subseteq \exp _{k} X$ is compact in $\exp _{k} X$ then $\bigcup C=\bigcup_{\alpha \in C} \alpha$ is compact in $X$.

Proof. Given an open cover $\mathcal{O}$ of $\bigcup C$, the set

$$
\mathcal{O}^{\prime}=\left\{\exp _{k} \cup O \mid O \subseteq \mathcal{O} \text { finite }\right\}
$$

is an open cover of $C$. Extracting a finite subcover

$$
\left\{\exp _{k} \cup O_{1}, \ldots, \exp _{k} \cup O_{m}\right\}
$$

from $\mathcal{O}^{\prime}$ we obtain a finite subcover $O_{1} \cup \cdots \cup O_{m}$ of $\mathcal{O}$.

Since compact subspaces of cell complexes lie in finite subcomplexes we have immediately:

Corollary 1. If $X$ is a cell complex and $C \subseteq \exp _{k} X$ is compact, then $C \subseteq \exp _{k} A$ for some finite subcomplex $A$ of $X$.

Proof of Theorem 1 for $X$ infinite. Let $[\phi] \in \pi_{i}\left(\exp _{k} X,\{v\}\right)$ for some vertex $v$ of $X$ and $i \leq k-2$. By Corollary $1 \phi\left(S^{i}\right)$ lies in $\exp _{k} A$ for some finite subcomplex $A$ of $X$, and we may take $A$ to be connected since $X$ is. By the finite case of the theorem $[\phi]$ is trivial in $\pi_{i}\left(\exp _{k} A,\{v\}\right)$, and so in $\pi_{i}\left(\exp _{k} X,\{v\}\right)$ also.

\section{Discussion}

Theorem 1 is consistent with the following conjecture on the finite subset spaces of cell complexes, as the theorem follows from the conjecture together with Handel's inclusion result. We restrict our attention to complexes with a single vertex in each component, with no loss of generality up to homotopy.

Conjecture. Let $X$ be a finite $n$-complex with c components, each containing a single vertex. Then $\exp _{k} X$ has a cell structure obtained from $\exp _{k-1} X$ by adding cells of dimensions $k-c \leq i \leq n k$. 
The conjecture is true in the connected case for $n=1$ by Lemma 1 of $[\mathbf{6}]$, and for $n=2$ by Theorem 6 of [7]. To see that it implies Theorem 1 note that it implies that the homotopy groups of $\exp _{k} X$ stabilise as $k$ increases. By Handel's result the stable groups must be zero when $X$ is connected, and careful attention to the point at which the stabilisation occurs gives the bound in the theorem.

Jacob Mostovoy (private communication) has indicated that finite subset spaces of cell complexes may be shown to have cell structures using the machinery of simplicial sets, which is described in [2] (E.B. Curtis) or [4] (J.P. May). Given a simplicial set $K$ we let $\exp _{j} K$ be the simplicial set whose $n$-simplices are subsets of size at most $j$ of the $n$-simplices of $K$, and whose face and degeneracy operators are the face and degeneracy operators of $K$ acting elementwise. Then if $X$ is the geometric realisation of $K$, $\exp _{j} X$ will be the geometric realisation of $\exp _{j} K$, showing that triangulated spaces have triangulated finite subset spaces. However, the triangulations obtained in this way apparently do not satisfy the lower bound $k-c$ needed to prove Theorem 1. This bound is motivated by the form of the lexicographic cell structures of [7] and is arrived at as follows: we suppose that $\exp _{k} X$ has a cell structure such that for each open cell $e$ of $X$ the set map

$$
\exp _{k} X \rightarrow \mathbf{N}: \Lambda \mapsto|\Lambda \cap e|
$$

is constant on each open cell $\sigma$ of $\exp _{k} X$, and moreover that the dimension of $\sigma$ is at least the common number of points in $X$ less the 0 -skeleton for $\Lambda \in \sigma$. In particular we suppose that the vertices of $\exp _{k} X$ can be chosen to be subsets of the vertices of $X$, without adding more through subdivision. If $X$ has a single vertex in each component then an open cell of $\exp _{k} X \backslash \exp _{k-1} X$ in such a cell structure would have dimension at least $k-c$.

Theorem 1 can be strengthened for simply connected complexes, and we expect that it can be strengthened further for $m$-connected cell complexes. With this in mind we prove the following theorem, showing it suffices to prove any strengthened result for wedges of spheres.

Theorem 2. Suppose that finite wedges of $(m+1)$-spheres have $r$-connected $k$-th finite subset spaces. Then $m$-connected cell complexes have $r$-connected $k$-th finite subset spaces also.

Proof. We simply adapt the inductive step of the proof of Theorem 1, replacing observation (2) with the following:

$\left(2^{\prime}\right)$ If $X$ is an $m$-connected finite $(n+1)$-complex, $n \geq m+1$, and $P \subseteq X$ lies in the open $(n+1)$-cells and intersects each in a nonempty finite set, then $X \backslash P$ has the homotopy type of an $m$-connected finite $n$-complex.

If $X$ is an $m$-connected finite cell complex then up to homotopy we may assume that the $(m+1)$-skeleton of $X$ is a finite wedge of $(m+1)$-spheres. 
The base for the induction is then given by hypothesis and the argument goes through exactly as before.

According to [7, Theorem 6], $\exp _{k} \bigvee_{n} S^{2}$ has a cell structure obtained from $\exp _{k-1} \bigvee_{n} S^{2}$ by adding cells in dimensions $k$ and higher, and using Handel's inclusion result and Theorem 2 we conclude that simply connected cell complexes have $(k-1)$-connected $k$-th finite subset spaces. More generally, the construction outlined in [7, Section 2.4] should yield cell structures for the finite subset spaces of wedges of spheres in which $\exp _{k} \bigvee_{n} S^{m+1}$ is obtained from $\exp _{k-1} \bigvee_{n} S^{m+1}$ by adding cells in dimensions $k+m-1$ and higher. Verifying the details of this construction would show that $m$-connected cell complexes have $(k+m-2)$-connected $k$-th finite subset spaces.

We conclude with an example showing the necessity of the connectedness hypothesis in Theorem 1. Consider the third finite subset space of a pair of circles, $\exp _{3}\left(S^{1} \amalg S^{1}\right)$. This has three connected components, two "pure" components consisting of subsets contained entirely in one or the other component circle, and a "mixed" component consisting of subsets meeting both. The two pure components are each homeomorphic to $S^{3}$ (Bott [1]; see also [5]), but the mixed component is formed by gluing two copies of $\exp _{2} S^{1} \times \exp _{1} S^{1} \cong \mathrm{Möb} \times S^{1}$ along their boundary. The gluing interchanges the roles of the boundary of the Möbius strip and the $S^{1}$ direction, and $\pi_{1}$ of the resulting three-manifold has presentation $\left\langle a, b \mid\left[a, b^{2}\right]=\left[a^{2}, b\right]=1\right\rangle$.

\section{References}

[1] R. Bott, On the third symmetric potency of $S_{1}$, Fund. Math., 39 (1952), 264-268, MR 0054954 (14,1003e), Zbl 0050.17801.

[2] E.B. Curtis, Simplicial homotopy theory, Advances in Mathematics, 6 (1971), 107-209, MR 0279808 (43 \#5529), Zbl 0225.55002.

[3] D. Handel, Some homotopy properties of spaces of finite subsets of topological spaces, Houston J. Math., 26(4) (2000), 747-764, MR 1823966 (2002d:55016), Zbl 0988.54015.

[4] J.P. May, Simplicial Objects in Algebraic Topology, Van Nostrand Mathematical Studies, 11, Van Nostrand, 1967; reprinted in 1992 by University of Chicago Press in the series Chicago Lectures in Mathematics, MR 1206474 (93m:55025), Zbl 0769.55001.

[5] C. Tuffley, Finite subset spaces of $S^{1}$, Alg. Geom. Topol., 2 (2002), 1119-1145, MR 1998017 (2004f:54008), Zbl 1015.55009.

[6] C. Tuffley, Finite subset spaces of graphs and punctured surfaces, Alg. Geom. Topol., 3 (2003), 873-904, MR 2012957 (2004i:55023), Zbl 1032.55014.

[7] C. Tuffley, Finite subset spaces of closed surfaces, math.GT/0311371.

\section{Department of Mathematics}

University of CALIFornia at Davis

DAVIS CA 95616-8633

E-mail address: tuffley@math.ucdavis.edu 